August, 1992

IASSNS-HEP-92/55

PUPT-1337

hep-th/9208076

\title{
On Cosmological String Backgrounds with Toroidal Isometries
}

\author{
Amit Giveon 1 \\ School of Natural Sciences, Institute for Advanced Study \\ Olden Lane, Princeton, NJ 08540, USA
}

Andrea Pasquinucci

Joseph Henry Laboratories, Department of Physics

Princeton University, Princeton, NJ 08544, USA

\begin{abstract}
A large class of cosmological solutions (of the Einstein equations) in string theory, in the presence of Maxwell fields, is obtained by $O(d, d)$ transformations of simple backgrounds with $d$ toroidal isometries. In all the examples in which we find a (closed) expanding universe, such that the universe admits a smooth, complete initial value hypersurface, a naked singularity may form only at the time when the universe collapses. The discrete symmetry group $O(d, d, Z)$ identifies different cosmological solutions with a background corresponding to a (relatively) simple CFT, and therefore, may be useful in understanding the properties of naked singularities in string theory.
\end{abstract}

\footnotetext{
${ }^{1}$ e-mail address: GIVEON@IASSNS.bitnet

Address after September 15: Racah Inst. of Physics, The Hebrew University, Jerusalem 91904, ISRAEL.

e-mail: GIVEON@HUJIVMS.bitnet
} 
To find realistic cosmological solutions in string theory, one represents a classical solution by $M \times K$, where $M$ is a $2-d$ conformal field theory (CFT) with a four dimensional space-time, and with a central charge $c=4$, and $K$ is some internal space represented also by a CFT. Moreover, though the possible formation of singularities is one of the questions of interest, we do not want the singularities to be "built in" in the initial conditions. We want $M$ to admit a smooth, complete initial value hypersurfaces.

In ref. [1], Nappi and Witten presented a CFT that describes a closed expanding universe in string theory. This universe is a cosmological solution with two toroidal isometries, namely, the background $M$ is independent of two coordinates.

In this paper, we use the method of $O(d, d)$ transformations [2, 3] in order to generate a large class of cosmological solutions in string theory. In particular, the solutions of [1] are just a one parameter sub-family, of the backgrounds obtained by $O(2,2)$ transformations of (a suitable analytic continuation of) a product of two $D=2$ black holes 4 . Moreover, when the internal space $K$ also has toroidal isometries, one can turn on non-trivial gauge fields. In the examples we discuss, we find an anisotropic expanding and contracting closed universe, in the presence of Maxwell fields. The topology of a spatial slice is that of a three sphere.

In the family of solutions we study, a naked singularity can appear only at the initial time, when the volume of the universe vanishes, and/or at the time when the universe recollapses. The cosmic censorship is therefore protected, within a large class of anisotropic cosmological solutions.

We begin by establishing our notation following [3]. The two dimensional action describing a string propagating in a (curved) background (of a metric, an antisymmetric tensor, and a dilaton) in $D$-dimensions, that is independent of $d$ coordinates is

$$
\begin{aligned}
S & =\frac{1}{2 \pi} \int d^{2} z\left[\Sigma_{I J}(x) \partial X^{I} \bar{\partial} X^{J}-\frac{1}{4} \phi(x) R^{(2)}\right] \\
& =\frac{1}{2 \pi} \int d^{2} z\left[E_{i j}(x) \partial \theta^{i} \bar{\partial} \theta^{j}+F_{i a}^{2}(x) \partial \theta^{i} \bar{\partial} x^{a}+F_{a i}^{1}(x) \partial x^{a} \bar{\partial} \theta^{i}\right.
\end{aligned}
$$




$$
\left.+F_{a b}(x) \partial x^{a} \bar{\partial} x^{b}-\frac{1}{4} \phi(x) R^{(2)}\right]
$$

where

$$
\left\{X^{I}\right\}_{I=1 \ldots D}=\left\{\theta^{i}, x^{a}\right\}_{i=1 \ldots d, a=d+1 \ldots D}, \quad \theta^{i} \equiv \theta^{i}+2 \pi
$$

and

$$
\Sigma_{I J}=G_{I J}+B_{I J}=\left(\begin{array}{ll}
E_{i j} & F_{i b}^{2} \\
F_{a j}^{1} & F_{a b}
\end{array}\right) .
$$

$G_{I J}$ and $B_{I J}$ are the symmetric and antisymmetric parts of $\Sigma_{I J}$, respectively. The dilaton $\phi$ in (11) is coupled to the world-sheet curvature.

The background $(\Sigma(x), \phi(x))$ is independent of the $d$ coordinates $\theta^{i}$. If the background $(\Sigma(x), \phi(x))$ corresponds to a $2-d$ CFT, it can be transformed to new conformal backgrounds by the action of a group isomorphic to $O(d, d, R)$. The proof is given in ref. [3] 2. The action of $O(d, d)$ on the background (3)

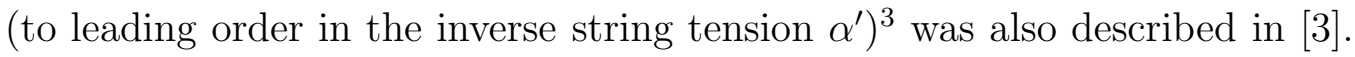
In this note we discuss examples for which $F^{1}=F^{2}=0$. In this case only the block $E_{i j}$ in (3) transforms under $O(d, d)$, while the block $F_{a b}$ is invariant. For the general case we refer the reader to ref. [3].

Next we describe the action of $O(d, d)$ on $E$ following [6]. The group $O(d, d)$ can be represented as $2 d \times 2 d$ dimensional matrices $g$ preserving the bilinear form $J$ :

$$
g=\left(\begin{array}{ll}
a & b \\
c & d
\end{array}\right), \quad J=\left(\begin{array}{ll}
0 & I \\
I & 0
\end{array}\right),
$$

where $a, b, c, d, I$ are $d \times d$ matrices, and

$$
g^{t} J g=J
$$

We define the action of $g$ on $E$ by fractional linear transformations:

$$
g(E)=E^{\prime}=(a E+b)(c E+d)^{-1} .
$$

\footnotetext{
2 The emphasize in ref. [3] is on the discrete sub-group $O(d, d, Z)$, but the statement is true for the full $O(d, d, R)$.

3 For simplicity, in (11) and in the rest of this paper we set $\alpha^{\prime}=\frac{1}{2}$. (For the insertion of $\alpha^{\prime}$ factors see for example [5].)
} 
The dilaton transformation is

$$
g(\phi)=\phi^{\prime}=\phi+\frac{1}{2} \log \left(\frac{\operatorname{det} G_{i j}}{\operatorname{det} G_{i j}^{\prime}}\right),
$$

where $G_{i j}\left(G_{i j}^{\prime}\right)$ is the symmetric part of $E_{i j}\left(E_{i j}^{\prime}\right)$.

If $(E, \phi)$ correspond to (a leading order in $\alpha^{\prime}$ of) a conformally exact background, then $\left(E^{\prime}, \phi^{\prime}\right)$ are the leading order in $\alpha^{\prime}$ of a conformally exact background as well. In particular, the low-energy effective action is invariant under $O(d, d)$ transformations [6, 2]. In other words, if $(G, B, \phi)$ solve the $\beta=0$ equations to leading order, so do $\left(G^{\prime}, B^{\prime}, \phi^{\prime}\right)$. One can therefore use the $O(d, d)$ transformations in order to generate new solutions to Einstein gravity coupled to matter, starting with a known solution with $d$ isometries [2].

We start to construct new solutions by exploiting the toroidal isometries of the space-time background $M$. Consider the $4-D$ line element

$$
d \mathbf{s}^{2}=-d t^{2}+d s^{2}+g(t)^{2} d \theta_{1}^{2}+f(s)^{2} d \theta_{2}^{2} .
$$

We want this background to correspond to a CFT.f To leading order in $\alpha^{\prime}$ there are four nontrivial possibilities for $g(t)$ and four possibilities for $f(s)$, namely, $g(t)=\tan (t), \cot (t), \tanh (t)$ or $\operatorname{coth}(t)$, and $f(s)=\tan (s), \cot (s), \tanh (s)$ or $\operatorname{coth}(s)$. T The dilaton is then given by

$$
\phi(s, t)=\phi_{0}+\log \left(\bar{f}(s)^{2} \bar{g}(t)^{2}\right)
$$

where $\phi_{0}$ is a constant and $\bar{g}(t)=\cos (t), \sin (t), \cosh (t)$ or $\sinh (t), \bar{f}(s)=$ $\cos (s), \sin (s), \cosh (s)$ or $\sinh (s)$, respectively.

The background in (8),(9) is a $D=4$ curved background that is independent of $d=2$ coordinates. It has one time-like coordinate $t$, and three space-like coordinates. The background is time dependent, and therefore, it

4 More precisely, we should start with $d \mathbf{s}^{2}=k\left(-d t^{2}+g(t)^{2} d \theta_{1}^{2}\right)+k^{\prime}\left(d s^{2}+f(s)^{2} d \theta_{2}^{2}\right)$, and choose $k$ and $k^{\prime}$ such that $c=4$. To leading order in $\alpha^{\prime}$ this condition is $k=k^{\prime}$, and for simplicity we take $k=k^{\prime}=1$.

5 All these possibilities correspond to the exact CFT given by a direct product of two cosets $\frac{G}{H}$, where $G$ is either $S U(2)$ or $S L(2)$ and $H$ is either $U(1)$ or $\mathbf{R}$. 
describes a cosmological solution to Einstein equations. The $2 \times 2$ matrix $E$ is

$$
E(s, t)=\left(\begin{array}{cc}
g(t)^{2} & 0 \\
0 & f(s)^{2}
\end{array}\right) .
$$

We can now generate new cosmological solutions acting on $(E, \phi)$ in (10), (9) with $O(2,2)$ transformations.

Next we discuss a particular one parameter sub-family of rotations generating the solutions of [1]. By transforming $E$ and $\phi$ with the group element $g_{b} \in O(2,2)$ (where $b$ is an arbitrary real number):

$$
g_{b}=\left(\begin{array}{cc}
0 & I \\
I & 0
\end{array}\right)\left(\begin{array}{cc}
I & b \Theta \\
0 & I
\end{array}\right)
$$

where

$$
I=\left(\begin{array}{ll}
1 & 0 \\
0 & 1
\end{array}\right), \quad \Theta=\left(\begin{array}{cc}
0 & 1 \\
-1 & 0
\end{array}\right)
$$

one finds that the new background matrix, $E_{b}^{\prime}$, is given by adding a constant antisymmetric background $\left(\begin{array}{cc}0 & b \\ -b & 0\end{array}\right)$ to $E$, and then using duality [7] to invert the background matrix, namely,

$$
g_{b}(E)=E_{b}^{\prime}=(E+b \Theta)^{-1}=\frac{1}{f(s)^{2} g(t)^{2}+b^{2}}\left(\begin{array}{cc}
f(s)^{2} & -b \\
b & g(t)^{2}
\end{array}\right) .
$$

From eq. (17) one gets for the new dilaton

$$
\phi^{\prime}(s, t)=\phi_{0}+\log \left[\bar{f}(s)^{2} \bar{g}(t)^{2}\left(f(s)^{2} g(t)^{2}+b^{2}\right)\right] .
$$

When

$$
\begin{array}{ll}
f(s)=\tan (s) \quad, \quad & g(t)=\cot (t), \\
\bar{f}(s)=\cos (s) \quad, \quad & \bar{g}(t)=\sin (t),
\end{array}
$$

we recover the solution discussed by Nappi and Witten in [1]. [1 In this case the conformal background corresponds to a closed expanding universe.

6 To compare with [1] one should use $\sin \alpha=\frac{1-b^{2}}{1+b^{2}}$, reintroduce $k$ and $k^{\prime}$, and take $k=k^{\prime}$ very large such that the maximal size of the universe is of order $k$, and the central charge of $M$ is $c=4$ (to leading order in $1 / k$ ). 
We will now consider the case in which the internal space $K$ also has toroidal isometries, and by rotating on non-trivial elements of the background matrix interpolating between the space-time $M$ and the internal space $K$, we will turn on the gauge fields.

The action (11) can be generalized adding internal degrees of freedom, i.e. extra compactified coordinates $Y^{A}$. (The coordinates $Y^{A}$ can be regarded as either the internal coordinates of a bosonic string or the extra coordinates of a heterotic string). Following ref. [3], we start from the action

$$
S=\frac{1}{2 \pi} \int d^{2} z\left[\Sigma_{I J}(x) \partial X^{I} \bar{\partial} X^{J}+E_{A B} \partial Y^{A} \bar{\partial} Y^{B}-\frac{1}{4} \phi(x) R^{(2)}\right],
$$

where the space-time coordinates $X^{I}$ are described in (2), the blocks structure of $\Sigma_{I J}$ is given in (3), and $E_{A B}$ is a constant matrix, $A, B=1, \ldots, d_{\text {int }}$. The background fields $\Sigma_{I J}(x), \phi(x), E_{A B}$ are independent of the coordinates $\left(\theta^{i}, Y^{A}\right)$.

We choose the initial structure of the background as before, namely, the block $E_{i j}$ is again given by eq. (10), $F^{1}=F^{2}=0$, and $F=\left(\begin{array}{cc}-1 & 0 \\ 0 & 1\end{array}\right)$. It is now possible to make a more general rotation using the group $O\left(2,2+d_{\text {int }}\right) \subset$ $O\left(2+d_{\text {int }}, 2+d_{\text {int }}\right)$ acting by the fractional linear transformations (6) on the $\left(2+d_{i n t}\right) \times\left(2+d_{\text {int }}\right)$ matrix

$$
\Xi=\left(\begin{array}{cc}
E_{i j} & 0 \\
0 & E_{A B}
\end{array}\right)
$$

such that

$$
\Xi^{\prime}=\left(\begin{array}{cc}
E_{i j}^{\prime}+A_{i A}\left(G^{-1}\right)^{A B} A_{j B} & 2 A_{i A} \\
0 & E_{A B}
\end{array}\right)
$$

where $\left(G^{-1}\right)^{A B}$ is the inverse of $G_{A B}$ defined by $E_{A B}=G_{A B}+B_{A B}$. The structure of $\Xi^{\prime}$ is such that $E_{i j}^{\prime}=G_{i j}^{\prime}+B_{i j}^{\prime}$ gives the correct metric after a dimensional reduction from $4+d_{\text {int }}$ dimensions to four space-time dimensions. The structure of the second line in the matrix $\Xi^{\prime}$ allows the internal coordinates to be the extra coordinates of a heterotic string.

The new background in (18) corresponds to the following (curved) bosonic or heterotic two dimensional action (neglecting worldsheet fermions in the 
heterotic case):

$S^{\prime}=\frac{1}{2 \pi} \int d^{2} z\left[\Sigma_{I J}^{\prime}(x) \partial X^{I} \bar{\partial} X^{J}+2 A_{I A}(x) \partial X^{I} \bar{\partial} Y^{A}+E_{A B} \partial Y^{A} \bar{\partial} Y^{B}-\frac{1}{4} \phi^{\prime}(x) R^{(2)}\right]$.

Both the starting background and the new metric, antisymmetric tensor, dilaton and gauge fields are solutions of the equations of motion derived from the effective action (see [5] for a review)

$$
S_{e f f}=\int d^{4} X \sqrt{-G} e^{\phi}\left[R^{(4)}+(\nabla \phi)^{2}-\frac{1}{12} H^{2}-\frac{1}{4} \operatorname{Tr} F^{2}\right] .
$$

(The starting background has $H_{\mu \nu \rho}=F_{\mu \nu}=0$.)

For simplicity, we now consider the case in which $d_{i n t}=1$, i.e. we start from a five dimensional background where the fifth coordinate is compact and by an $O(2,3) \subset O(3,3)$ rotation we will introduce a Maxwell field $A_{i}$. (The discussion can be easily generalized to the internal dimension $d_{\text {int }}$ that is needed for criticality.)

We fix $E_{A B}=1$ and we rotate $\Xi$ by the $g_{a, b, c} \in O(2,3) \subset O(3,3)$ matrix

$$
g_{a, b, c}=\left(\begin{array}{cc}
0 & I \\
I & 0
\end{array}\right)\left(\begin{array}{cc}
I & \Theta \\
0 & I
\end{array}\right)\left(\begin{array}{cc}
A & 0 \\
0 & \left(A^{t}\right)^{-1}
\end{array}\right)
$$

where

$$
\Theta=\left(\begin{array}{ccc}
0 & b & c \\
-b & 0 & a \\
-c & -a & 0
\end{array}\right), \quad A=\left(\begin{array}{ccc}
1 & 0 & c \\
0 & 1 & a \\
0 & 0 & 1
\end{array}\right)
$$

$I$ is the $3 \times 3$ identity matrix and $a, b, c$ are arbitrary real numbers.

After the rotation, the non-zero components of the metric $G_{I J}^{\prime}{ }^{7}$, antisymmetric tensor $B_{I J}^{\prime}$, gauge field $A_{I}$ and dilaton $\phi^{\prime}$, are:

$$
\begin{aligned}
& G_{t t}^{\prime}=-1 \quad G_{s s}^{\prime}=1 \\
& G_{\theta_{1} \theta_{1}}^{\prime}=\frac{1}{\Delta^{2}}\left[g(t)^{2}\left(a^{2}+f(s)^{2}\right)^{2}+f(s)^{2}(a c+b)^{2}\right] \\
& G_{\theta_{1} \theta_{2}}^{\prime}=\frac{1}{\Delta^{2}}\left[b\left(a^{2} g(t)^{2}-c^{2} f(s)^{2}\right)-a c\left(a^{2} g(t)^{2}+c^{2} f(s)^{2}+2 f(s)^{2} g(t)^{2}\right)\right]
\end{aligned}
$$

\footnotetext{
7 Recall that in eq. (19) $\Sigma_{I J}^{\prime}=G_{I J}^{\prime}+B_{I J}^{\prime}$.
} 


$$
\begin{array}{ll}
G_{\theta_{2} \theta_{2}}^{\prime}=\frac{1}{\Delta^{2}}\left[f(s)^{2}\left(c^{2}+g(t)^{2}\right)^{2}+g(t)^{2}(a c-b)^{2}\right] \\
A_{\theta_{1}}=\frac{1}{\Delta}\left[a b-c f(s)^{2}\right] & A_{\theta_{2}}=\frac{1}{\Delta}\left[-b c-a g(t)^{2}\right] \\
B_{\theta_{1} \theta_{2}}^{\prime}=-\frac{b}{\Delta} & \phi^{\prime}=\phi_{0}+\log \left[\bar{f}(s)^{2} \bar{g}(t)^{2} \cdot \Delta\right] \\
\operatorname{det}\left[-G^{\prime}\right]=\frac{g(t)^{2} f(s)^{2}}{\Delta^{2}} &
\end{array}
$$

where

$$
\Delta=b^{2}+a^{2} g(t)^{2}+c^{2} f(s)^{2}+g(t)^{2} f(s)^{2} .
$$

Obviously, for $a=c=0$, one gets back the space-time solutions in (13) and (14).

It is also possible to make a Weyl rescaling to bring the effective action (20) to the Einstein form

$$
S_{\text {eff }}^{E}=\int d^{4} X \sqrt{-G^{E}}\left[R^{E(4)}-\frac{1}{2}(\nabla \phi)^{2}-\frac{1}{12} e^{2 \phi} H^{2}-\frac{1}{4} e^{\phi} \operatorname{Tr} F^{2}\right]
$$

where the metric $G_{\mu \nu}^{E}=e^{\phi} G_{\mu \nu}$ is used to contract the indices.

Notice that when $f$ and $g$ are given by eq. (15) (for example) one has

$$
\begin{aligned}
\phi^{\prime}=\phi_{0}+\log [ & b^{2} \sin ^{2}(t) \cos ^{2}(s)+a^{2} \cos ^{2}(t) \cos ^{2}(s) \\
& \left.+c^{2} \sin ^{2}(t) \sin ^{2}(s)+\cos ^{2}(t) \sin ^{2}(s)\right] .
\end{aligned}
$$

For non-vanishing parameters $a, b$ and $c$, the dilaton is finite for any $s, t$ (and therefore, Weyl rescaling to the Einstein form does not change the properties of the universe described above). For $a=0$ the dilaton diverges at $(t=0, s=0)$, for $c=0$ it diverges at $(t=\pi / 2, s=\pi / 2)$ and for $b=0$ it diverges at $(t=\pi / 2, s=0)(t, s \in[0, \pi / 2])$. These divergences appear when a curvature singularity is formed.

We will now discuss briefly the target space properties of some of the models just presented. From eq.(23), we see that the universe described by 
that class of solutions has zero volume at $t=0$ (this is because $g(t=0)=0$ or $\infty$ for all possible $g$ 's). Depending on the choices of the functions $f, g$ and the parameters $a, b, c$, the universe can then be an expanding and contracting closed universe, in some cases collapsing at the end, or an expanding open universe. (We will not consider the models which do not have a smooth, complete initial value hypersurface. An example of models with "built in" singularities in the initial conditions is given when $a=b=0, c \neq 0$ and the functions $f$ and $g$ given by eq. (15).)

Consider again the case when the functions $f$ and $g$ are given by eq. (15) (with $b$ and $c$ not both zero). For this example, the backgrounds in (23) describe an anisotropic expanding and contracting universe, in the presence of an antisymmetric tensor, a Maxwell field, and a dilaton. The topology of a spatial slice is $S^{3}$, the three sphere. The universe recollapses at the time $t=\frac{\pi}{2}$.

When $b \neq 0$, this universe is a modification of the Nappi-Witten solution [1] in the presence of the Maxwell field $\left(A_{\theta_{1}}(s, t), A_{\theta_{2}}(s, t)\right)$ given in (23). The universe expands and collapses in the presence of electro-magnetic currents. (There is no electric charge).

The case $b=0(c \neq 0)$ is particularly interesting. In this case we find a classical solution to dilaton gravity with a Maxwell field (given by the action (20) with $H=0$, namely, without the presence of the antisymmetric background that vanishes for $b=0$ ). This solution still corresponds to a closed expanding universe as described above.

Next we discuss the formation of singularities in the universe described above. There are different cases depending on the parameters $a, b$, and $c$. For $a=0$ the universe is singular at the 'big bang', namely, when $t=0$ a singularity is formed at $s=0$. For $b=0(c=0)$, a naked singularity is about to form at $s=0(s=\pi / 2)$ at the time $t=\pi / 2$, when the universe recollapses. (For non-vanishing parameters $a, b$ and $c$ there are no singularities). Therefore, a naked singularity may form only at the times when the universe collapses. 
Finally, we point out briefly some consequences of the generalized target space duality. It was proven in ref. 3] that the elements of $O(d, d, Z)$ are discrete symmetries of the space of curved string backgrounds that are independent of $d$ coordinates. These discrete symmetries relate vacua with geometries that in general are radically different, but that correspond to the same $2-d$ conformal field theory (CFT).

In the examples we discussed, the matrix $g_{b}$ in eq. (11) $\left(g_{a, b, c}\right.$ in eq. (21)) is in $O(2,2, Z)(O(3,3, Z))$ if $b \in Z(a, b, c \in Z)$. For example, as a consequence, all the Nappi-Witten solutions [1] with $\sin \alpha=\frac{1-b^{2}}{1+b^{2}}, b \in Z$ are equivalent as CFTs, and in particular, they are all equivalent to the $b=0$ case, for which the background is a direct product of (a suitable analytic continuation) of two $D=2$ black hole solutions [4]. Moreover, all the solutions (23) with $a, b, c \in Z$ are also equivalent CFTs. Therefore, these closed expanding universes in the presence of electro-magnetic currents, are also equivalent as CFTs to the direct product of (a suitable analytic continuation) of two $D=2$ black hole solutions, and an extra coordinate compactified on a circle.

The discrete symmetries identify different cosmological solutions with a background corresponding to a (relatively) simple CFT. Therefore, target space dualities may be useful in understanding the properties of naked singularities in string theory.

\section{Acknowledgements}

We thank K. Bardakci, C. Nappi, and E. Witten for useful discussions. We would like to acknowledge the hospitality of the Theoretical Physics Group at Lawrence Berkeley Laboratory where part of this work was done. The work of A.G. is supported in part by DOE grant no. DE-FG02-90ER40542. The research of A.P. is supported by an INFN fellowship and partially by the NSF grant PHY90-21984.

\footnotetext{
8 This was pointed out for stringy black hole singularities in [8].
} 


\section{References}

[1] C.R. Nappi and E. Witten, "A closed, expanding universe in string theory", preprint IASSNS-HEP-92/38, 1992.

[2] K.A. Meissner and G. Veneziano, Phys. Lett. 267 (1991) 33; M. Gasperini, J. Maharana and G. Veneziano, Phys. Lett. 272 (1991) 277.

[3] A. Giveon and M. Rocek, Nucl. Phys. B380 (1992) 128.

[4] E. Witten, Phys. Rev. D44 (1991) 314.

[5] M.B. Green, J.H. Schwarz and E. Witten, 'Superstring theory' (Cambridge Univ. Press, Cambridge, 1987).

[6] A. Giveon, N. Malkin and E. Rabinovici, Phys. Lett. B238 (1990) 57.

[7] A. Giveon, E. Rabinovici and G. Veneziano, Nucl. Phys. B322 (1989) 167; A. Shapere and F. Wilzcek, Nucl. Phys. B320 (1989) 669.

[8] A. Giveon, Mod. Phys. Lett. A6 (1991) 2843; R. Dijkgraaf, E. Verlinde and H. Verlinde, Nucl. Phys. B371 (1992) 269. 